\title{
AVALIAÇÃO DA ADESÃO AO TRATAMENTO ANTIRRETROVIRAL DE PESSOAS VIVENDO COM HIV
}

\section{Marcelo Ribeiro Primeira}

Enfermeiro. Discente do Curso de Mestrado do Programa de Pós-Graduação da Universidade Federal de Santa Maria (UFSM/RS), Brasil.

\section{Érika Eberlline Pacheco dos Santos}

Enfermeira. Mestra em Enfermagem. Docente da Universidade do Oeste de Santa Catarina (UNOESC), Campus São Miguel do Oeste, Brasil.

\section{Samuel Spiegelberg Züge}

Enfermeiro. Discente do curso de Doutorado do Programa de Pós-Graduação em Enfermagem da Universidade Federal de Santa Maria (UFSM/RS). Docente da Universidade do Oeste Catarinense e Colaborador da Universidade do Estado de Santa Catarina, Brasil.

\section{Tânia Solange Bosi de Souza Magnago}

Enfermeira. Doutora em Enfermagem, Setor de Vigilância em Saúde e Segurança do Paciente (HUSM/ EBSERH) Departamento de Enfermagem (PPGEnf/ CCS) da Universidade Federal de Santa Maria (UFSM/ RS), Brasil.

\section{Cristiane Cardoso de Paula}

Enfermeira. Doutora em Enfermagem Departamento de Enfermagem (PPGEn/CCS) na Universidade Federal de Santa Maria (UFSM/RS), Brasil.

\section{Stela Maris de Mello Padoin}

Enfermeira. Doutora em Enfermagem Departamento de Enfermagem (PPGEnf/CCS) na Universidade Federal de Santa Maria (UFSM/RS), Brasil.
RESUMO: Avaliar a adesão ao tratamento antirretroviral de pessoas que vivem com HIV através do "Cuestionario para la Evaluación de la Adhesión al Tratamiento Antiretroviral" (CEAT-VIH), de forma a descrever seus itens a partir da correlação entres as questões deste instrumento. Estudo transversal, realizado em um Hospital Universitário do Rio Grande do Sul no primeiro semestre de 2012 com 179 pessoas em tratamento para o HIV. Foram realizadas análises estatísticas descritivas e de correlação entre os itens do questionário. Avaliação da adesão: 46,4\% baixa/insuficiente, $36,9 \%$ boa/adequada e 16,8\% estrita. Correlações: deixar de tomar a medicação relaciona-se aos fatos de se sentir melhor/pior, ou sentir-se triste/deprimido. Seguir a prescrição médica está relacionado com a autoavaliação em relação à ingesta dos medicamentos. A baixa/inadequada adesão legitima o uso do CEAT-VIH enquanto ferramenta a ser utilizada pela enfermagem junto à equipe multiprofissional no planejamento do cuidado e intervenção nas situações que interferem na adesão.

PALAVRAS-CHAVE: Síndrome da imunodeficiência adquirida; HIV; Doenças sexualmente transmissíveis; Adesão à medicação; Terapia antirretroviral de alta atividade; Enfermagem.

\section{ASSESSMENT OF ADHESION OF ANTI-RETROVIRAL TREATMENT IN PEOPLE WITH HIV}

\begin{abstract}
Current analysis assesses adhesion to anti-retroviral treatment in HIV patients by "Cuestionario para la Evaluación de la Adbesión al Tratamiento Antiretroviral" (CEAT-VIH), for the description of items by co-relation between the instrument's issues. The transversal study was performed at the Hospital Universitário do Rio Grande do Sul during the first semester of 2012, comprising 179 people undergoing HIV treatment. Descriptive statistical and corelation analyses between the questionnaire's items were performed. Adhesion evaluation was as follows: $46.4 \%$ low/insufficient; $36.9 \%$ good/adequate; $16.8 \%$ strict. Co-relationship: not taking medicine is related to feeling better/worse, or feeling bad, depressed. Following the doctor's prescription is related to self-evaluation with regard to taking medicines. Low/inadequate adhesion triggers the use of CEAT-VIH as a tool that may be used by nurses plus a multiprofissional team in the planning of care and intervention in situations that impair adhesion.
\end{abstract}

KEY WORDS: Acquired immunodeficiency syndrome; HIV; Sexually transmitted diseases; Adhesion to medication; High activity antiretroviral therapy; Nursing. 


\section{INTRODUÇÃO}

A epidemia causada pelo Vírus da Imunodeficiência Humana (HIV) é reconhecida como um problema de saúde pública tanto pelo seu impacto individual, quanto pelo seu impacto na sociedade relacionado, também, ao seu potencial epidêmico. Portanto, desde o início de sua epidemia são necessárias políticas públicas de saúde e estratégias por parte dos governos para conter os agravos decorrentes da infecção pelo HIV ${ }^{1,2}$.

Aliado à dispensação gratuita dos medicamentos antirretrovirais (ARV), outra estratégia utilizada é o diagnóstico e o tratamento precoce àqueles infectados pelo HIV. O diagnóstico precoce, promovido pela disponibilidade de testes rápidos na Atenção Primária à Saúde, seguido da terapia antirretroviral (TARV) eficaz, eleva os níveis de sobrevida, diminui a morbidade e mortalidade pela Síndrome da Imunodeficiência Adquirida (AIDS) - estágio avançado da infecção pelo HIV - e diminui a incidência de novos casos relacionados ao vírus ${ }^{3,4}$. Dessa forma, com diagnóstico e tratamento precoces, a infecção causada pelo HIV descaracteriza-se como doença letal e passa a ser considerada como uma condição crônica e de potencial controle ${ }^{5}$.

O controle do HIV e a manutenção da saúde são promovidos pelo uso contínuo e adequado da TARV, esta terá efetividade quando a ingesta dos medicamentos se der conforme a prescrição, determinando sua adesão ao tratamento. Para uma adesão satisfatória, a utilização dos medicamentos ARV deve ser mais próxima possível da prescrição fornecida pela equipe de saúde, de forma a cumprir horários, doses e demais indicações prescritas ${ }^{6}$. No entanto, a adesão é um dos maiores desafios durante o tratamento para o HIV, pois envolve não somente o uso dos ARV, como também fatores comportamentais e relacionados ao sistema de saúde?.

A adesão também é um processo colaborativo vinculado ao princípio da autonomia que implica na participação ativa da PVHIV no cuidado à sua saúde. Esta cooperação entre equipe e sujeito facilita a aceitação e a incorporação do esquema terapêutico no cotidiano de seu tratamento ${ }^{6}$.

A política de início imediato do uso dos ARV às PVHIV, considerando sua motivação pessoal, vai ao encontro do objeto deste estudo: adesão ao tratamento . $^{4}$ Consequentemente, a adesão se torna tema central quando refletimos acerca das políticas públicas de saúde relacionadas ao controle da epidemia do HIV, uma vez que com o diagnóstico e tratamento precoces será cada vez maior o número de PVHIV que irão iniciar seu tratamento.

Justifica-se este estudo por existirem desafios referentes às formas de monitoramento e avaliação da adesão, por não haver um padrão de procedimentos que garanta de forma fidedigna à medida de adesão, e pela maioria das avaliações contemplarem apenas a ingesta dos ARV. Desta forma, a fim de garantir a adesão, os profissionais, equipes e serviços de saúde necessitam tratar esta problemática como prioridade nas práticas assistenciais, além de buscar avaliação que contemple o comportamento da ingestão dos medicamentos, e também as dificuldades e barreiras percebidas pelas PVHIV para cumprir o tratamento ${ }^{8}$.

Assim, tem-se como objetivo: avaliar a adesão ao tratamento antirretroviral de pessoas que vivem com HIV por meio do "Cuestionario para la Evaluación de la Adhesión al Tratamiento Antiretroviral" (CEAT-VIH), de forma a descrever seus itens a partir da correlação entres as questões deste instrumento.

\section{METODOLOGIA}

Este estudo é caracterizado como de abordagem quantitativa, descritiva e delineamento transversal. O cenário para a coleta de dados foi um ambulatório de doenças infecciosas de um Hospital Universitário (HU) da região Centro-oeste do Estado do Rio Grande do Sul/Brasil. Este HU é referência nos níveis secundário e terciário de atenção à saúde, em média e alta complexidade para 32 municípios de abrangência da $4^{\mathrm{a}}$ Coordenadoria Regional de Saúde ( $\left.4^{\mathrm{a}} \mathrm{CRS}\right)$. O ambulatório referenciado, da mesma forma que o HU, também é referência regional para o atendimento de doenças infecciosas, incluindo a infecção pelo HIV e AIDS. O período da coleta de dados foi de janeiro a junho de 2012.

Foram incluídas no estudo PVHIV com 20 anos ou mais de idade, em TARV, e que realizassem 
acompanhamento ambulatorial no mínimo há três meses no serviço. Os critérios de exclusão compreenderam: pessoas com alguma limitação cognitiva e/ou mental (dificuldade de compreensão e/ou expressão verbal), àquelas em regime penitenciário por ferir o princípio da privacidade e mulheres em período gravídico-puerperal (pelo motivo de que durante o período gestação os antirretrovirais são utilizados como medida profilática da transmissão vertical do HIV e não como tratamento de sua situação de infecção, o que poderia ocasionar viés no estudo). Atendiam aos critérios de inclusão 432 pessoas, das quais 179 fizeram parte da amostra, com um erro amostral de 5,7\%, intervalo de confiança de $95 \%$ e proporção de $50 \%$.

Para a etapa de coleta de dados, a amostra foi alcançada por conveniência, ou seja, as pessoas foram convidadas a participar do estudo à medida que chegavam ao serviço, sendo aplicado o instrumento CEAT-VIH, que foi traduzido, adaptado e validado para a população brasileira ${ }^{8}$. A construção do banco de dados foi realizada no programa Epi-info ${ }^{\circledR}$ versão 3.5 com dupla digitação independente a fim de garantir a exatidão dos dados; estes foram analisados no programa SPSS versão 18.0.

A análise das variáveis sociais e demográficas e econômicas foi por meio de estatística descritiva, e contemplou as seguintes questões:

- sexo (masculino e feminino);

- faixa etária em anos (20 a 29, 30 a 39, 40 a 49,50 a 59 e mais de 59 anos);

- escolaridade (não estudou, estudou até ensino fundamental, estudou até o ensino médio, estudou até o ensino superior);

- filhos (nenhum filho, 01 filho, 02 a 04 filhos, 05 ou mais filhos);

- situação marital (convive com esposo(a)/ companheiro(a), vive sozinho(a));

- renda per capita da família $(\mathrm{n}=174)$ (até 02 salários mínimos, mais de 02 salários mínimos).

O CEAT-VIH é constituído por 20 itens que contemplam cinco dimensões: a conformidade com o tratamento; os fatores que modulam a adesão; a interação entre profissional e paciente; as crenças do paciente relacionadas ao esforço e o tempo de tratamento; e a avaliação da gravidade dos efeitos colaterais. A pontuação total do CEAT-VIH é obtida pela soma de todos os itens (valor mínimo possível: 17; e valor máximo possível: 89). Quanto maior a pontuação, maior o grau de adesão ao tratamento. O grau de adesão foi classificado entre "baixa/insuficiente" (pontuação < 77), "boa/adequada" (pontuação $\geq 77$ e $<83$ ) e "estrita" (pontuação $\geq 83$ ) .

A consistência interna do CEAT-VIH foi avaliada por meio do coeficiente Alpha de Cronbach $(0,782)$ e o teste de normalidade apontou uma distribuição assimétrica do CEAT-VIH, segundo teste Kolmogorov-Smirnov.

Foi realizada a correlação de Pearson entre os itens que compõem o CEAT-VIH. A correlação é interpretada como a relação que existe entre duas variáveis (itens) com o objetivo de apontar o grau de relação entre elas. O coeficiente de correlação (r) está compreendido entre $-1<\mathrm{r}<1$. Dessa forma, quando "r" expõe valores positivos, as variáveis estão diretamente relacionadas, da mesma forma que quando " $r$ " expõe valores negativos, as variáveis estão inversamente relacionadas. Os valores de interpretação do coeficiente de correlação são $(\mathrm{r}=1)$ correlação perfeita; $(0,80<\mathrm{r}<1)$ muito alta; $(0,60<\mathrm{r}$ $<0,80)$ alta; $(0,60<\mathrm{r}<0,40)$ moderada; $(0,20<\mathrm{r}<$ $0,40)$ baixa; $(0,10<\mathrm{r}<0,20)$ muito baixa; $(\mathrm{r}=0)$ nula $^{10}$.

Para as análises de correlação foi adotado o nível de confiança de $95 \%(p<0,01)$ e os valores de correlação $(r=<0,40)$.

Por fim, os aspectos éticos e metodológicos devem estar de acordo com as diretrizes estabelecidas na resolução 196/96 (vigente na época) e complementares do Conselho Nacional de Saúde, tendo aprovação do Comitê de Ética em Pesquisa da Universidade Federal de Santa Maria sob o número de processo 23081.015120/2011-15.

\section{RESULTADOS}

Das 179 PVHIV que compuseram a amostra, 50,8\% eram do sexo masculino, $36,3 \%$ tinham idade entre 40 e 49 anos, 52,5\% tinham estudado até o ensino fundamental, $42,4 \%$ tinham de dois a quatro filhos, $51,4 \%$ viviam sozinhos e 73,0\%, quanto à renda per capita, recebiam 
até dois salários mínimos (valor do salário mínimo em 2012: $\mathrm{R} \$ 622,00)$.

Conforme a soma das respostas do CEAT-VIH, a média da pontuação foi de 75,59 pontos, com desviopadrão de 7,68. Quanto ao grau de adesão, conforme pontuação, 46,4\% apresentaram adesão baixa/insuficiente, 36,9\% adesão boa/adequada e 16,8\% adesão estrita.

No Quadro 01 estão expostos, a partir dos itens do CEAT-VIH, os valores mais altos de interpretação encontrados neste estudo e que apresentaram correlação moderada e são diretamente relacionados, resultados utilizados para a discussão deste artigo.

Quadro 1. Coeficientes de correlação de Pearson moderada e diretamente relacionados segundo itens do CEAT-VIH. Interior do Rio Grande do Sul, 2012

\begin{tabular}{|c|c|c|c|c|c|c|c|}
\hline $\begin{array}{c}\text { Itens } \\
\text { CEAT- } \\
\text { VIH }\end{array}$ & 01 & 02 & 03 & 09 & 10 & 11 & 12 \\
\hline 02 & 0,511 & & & & & & \\
\hline 03 & 0,456 & 0,449 & & & & & \\
\hline 04 & 0,424 & 0,565 & 0,538 & & & & \\
\hline 10 & & & & 0,483 & & & \\
\hline 11 & & & & 0,435 & 0,418 & & \\
\hline 14 & & & & & 0,460 & 0,452 & \\
\hline 17 & & & & & & & 0,457 \\
\hline
\end{tabular}

Subsequentemente, a fim de contemplar um dos objetivos deste estudo, são descritos os itens que compõem o CEAT-VIH bem como os resultados de maior valor percentual dentre as opções. Estes itens serão expostos no decorrer do texto em tabelas distintas para facilitar a interpretação, tanto dos resultados relacionados à correlação já exibidos no Quadro 01, quanto àqueles que não apresentaram índices de correlação.

Na Tabela 01 são apresentados os coeficientes de correlação em todos seus itens. O item 01 "Deixou de tomar sua medicação alguma vez?" apresentou correlação com os itens 02,03 e 04 . Ou seja, deixar de tomar a medicação alguma vez está ligado aos fatos de se sentir melhor (item 02), se sentir pior (item 03) e sentir-se triste ou deprimido.
Tabela 1. Descrição dos itens (01 a 04) que compõem o CEATVIH, opção de maior frequência e desvio-padrão. Interior do Rio Grande do Sul, 2012

\begin{tabular}{lccc}
\hline \multicolumn{1}{c}{ Itens CEAT-VIH } & Opção & $\%$ & $\begin{array}{l}\text { Desvio- } \\
\text { padrão }\end{array}$ \\
\hline $\begin{array}{l}\text { 01 - Deixou de tomar sua } \\
\text { medicação alguma vez? }\end{array}$ & $\begin{array}{c}\text { Nenhuma } \\
\text { vez }\end{array}$ & 55,3 & 0,751 \\
$\begin{array}{l}\text { 02 - Se alguma vez sentiu-se } \\
\text { melhor, deixou de tomar sua } \\
\text { medicação? }\end{array}$ & $\begin{array}{c}\text { Nenhuma } \\
\text { vez }\end{array}$ & 77,1 & 0,764 \\
$\begin{array}{l}\text { 03 - Se alguma vez depois } \\
\text { de tomar sua medicação } \\
\text { sentiu-se pior, deixou de } \\
\text { tomá-la? }\end{array}$ & $\begin{array}{c}\text { Nenhuma } \\
\text { vez }\end{array}$ & 79,9 & 0,730 \\
$\begin{array}{l}\text { 04-Se alguma vez se sentiu } \\
\text { triste ou deprimido, deixou } \\
\text { de tomar sua medicação? }\end{array}$ & $\begin{array}{c}\text { Nenhuma } \\
\text { vez }\end{array}$ & 79,3 & 0,689 \\
\hline
\end{tabular}

$\mathrm{Na}$ Tabela 02, do quinto ao oitavo item, são exibidas as opções de maior valor percentual, e que não apresentarem alguma correlação.

Tabela 2. Descrição dos itens ( 05 a 08$)$ que compõem o CEATVIH, opção de maior porcentagem e desvio-padrão. Interior do Rio Grande do Sul, 2012

\begin{tabular}{lccc}
\hline \multicolumn{1}{c}{ Itens CEAT-VIH } & Opção & $\%$ & $\begin{array}{l}\text { Desvio } \\
\text { Padrão }\end{array}$ \\
\hline $\begin{array}{l}\text { 05 - Lembra-se que remédios } \\
\text { está tomando nesse } \\
\text { momento? }\end{array}$ & $\begin{array}{c}\text { Não } \\
\text { recorda } \\
\text { nenhum }\end{array}$ & 55,3 & 0,902 \\
$\begin{array}{l}06 \text { - Como é a relação que } \\
\text { mantém com o seu médico? }\end{array}$ & Boa & 81,0 & 0,785 \\
$\begin{array}{l}\text { 07 - Quanto esforço você faz } \\
\text { para seguir (cumprir) com o } \\
\text { seu tratamento? }\end{array}$ & Muito & 27,4 & 1,563 \\
$\begin{array}{l}08 \text { - Quanta informação você } \\
\text { tem sobre os medicamentos } \\
\text { que toma para o HIV? }\end{array}$ & Nada & 29,1 & 1,265 \\
\hline
\end{tabular}

Observam-se na Tabela 03 os itens do questionário do número 09 ao 17 e, dentre estes, os de número 09, 10, 11, 12, 14 e 17 apresentaram coeficientes de correlação e sua descrição é exibida nos parágrafos abaixo da tabela.

As questões "Quanto benefício pode lhe trazer o uso destes medicamentos?" (item 09) e "Como se sente em geral com o tratamento desde que começou a tomar seus remédios?" (Item 14), apresentaram correlação com os itens 10 e 11. Dessa forma, os resultados nos dizem que a avaliação do benefício do uso dos medicamentos 
(item 09) e a avaliação de como se sente em geral com o tratamento (item 14) estão vinculadas à avaliação da melhora da saúde desde que iniciou o uso dos medicamentos (item 10) e também com a estimativa da capacidade de seguir o tratamento (item 11).

Além disso, observa-se a correlação entre as perguntas dos itens 12 e 17, "Normalmente está acostumado a tomar a medicação na hora certa?", "Que avaliação tem de si mesmo com relação a toma dos remédios para o HIV?" respectivamente. Assim, constatase que o fato de estar acostumado a tomar a medicação na hora certa (item 12) está associado à avaliação que a PVHA tem de si em relação à ingesta de seus medicamentos e vice-versa.

Tabela 3. Descrição dos itens ( 09 a 17) que compõem o CEAT$\mathrm{VIH}$, opção de maior frequência e desvio-padrão. Interior do Rio Grande do Sul, 2012

\begin{tabular}{|c|c|c|c|}
\hline Itens CEAT-VIH & Opção & $\%$ & $\begin{array}{l}\text { Desvio- } \\
\text { padrão }\end{array}$ \\
\hline $\begin{array}{l}09-\text { Quanto benefício } \\
\text { pode lhe trazer o uso destes } \\
\text { medicamentos? }\end{array}$ & Muito & 75,4 & 0,728 \\
\hline $\begin{array}{l}10 \text { - Considera que sua saúde } \\
\text { melhorou desde que começou } \\
\text { a tomar os medicamentos para } \\
\text { o HIV? }\end{array}$ & Muito & 53,6 & 0,972 \\
\hline $\begin{array}{l}11 \text { - Até que ponto sente- } \\
\text { se capaz de seguir com o } \\
\text { tratamento? }\end{array}$ & Muito & 68,7 & 0,742 \\
\hline $\begin{array}{l}12 \text { - Normalmente está } \\
\text { acostumado a tomar a } \\
\text { medicação na hora certa? }\end{array}$ & $\begin{array}{c}\text { Sim, } \\
\text { sempre }\end{array}$ & 68,7 & 0,889 \\
\hline $\begin{array}{l}\text { 13-Quando os resultados dos } \\
\text { exames são bons, seu médico } \\
\text { costuma utilizá-los para lhe } \\
\text { dar ânimo e motivação para } \\
\text { seguir com o tratamento? }\end{array}$ & $\begin{array}{c}\text { Sim, } \\
\text { sempre }\end{array}$ & 78,2 & 0,965 \\
\hline $\begin{array}{l}14 \text { - Como se sente em geral } \\
\text { com o tratamento desde } \\
\text { que começou a tomar seus } \\
\text { remédios? }\end{array}$ & $\begin{array}{c}\text { Muito } \\
\text { satisfeito }\end{array}$ & 62,0 & 0,722 \\
\hline $\begin{array}{l}15 \text { - Como avalia a intensidade } \\
\text { dos efeitos colaterais } \\
\text { relacionados com o uso dos } \\
\text { medicamentos para o HIV? }\end{array}$ & $\begin{array}{c}\text { Nada } \\
\text { intensos }\end{array}$ & 41,3 & 1,408 \\
\hline
\end{tabular}

\begin{tabular}{l|ccc}
\multicolumn{1}{c}{ Itens CEAT-VIH } & Opção & $\%$ & $\begin{array}{c}\text { Desvio- } \\
\text { padrão }\end{array}$ \\
\hline $\begin{array}{l}\text { 16 - Quanto tempo acredita } \\
\text { que perde ocupando-se em } \\
\text { tomar seus remédios? }\end{array}$ & $\begin{array}{c}\text { Nada de } \\
\text { tempo }\end{array}$ & 68,7 & 0,849 \\
$\begin{array}{l}17-\text { Que avaliação tem de si } \\
\text { mesmo com relação a toma } \\
\text { dos remédios para o HIV? }\end{array}$ & $\begin{array}{c}\text { Muito } \\
\text { cumpridor }\end{array}$ & 60,3 & 0,959 \\
\hline
\end{tabular}

Por fim, na Tabela 04, são apresentados os últimos itens (18 ao 20) do instrumento com suas respectivas opções de maior valor percentual. Esses itens também não apresentaram correlação.

Tabela 4. Descrição dos itens (18 a 20) que compõem o CEATVIH, opção de maior frequência e desvio-padrão. Interior do Rio Grande do Sul, 2012

\begin{tabular}{lccc}
\hline \multicolumn{1}{c}{ Itens CEAT-VIH } & Opção & $\%$ & $\begin{array}{l}\text { Desvio- } \\
\text { padrão }\end{array}$ \\
\hline $\begin{array}{l}18 \text { - Quanta dificuldade tem } \\
\text { para tomar a medicação? }\end{array}$ & $\begin{array}{c}\text { Nenhuma } \\
\text { dificuldade }\end{array}$ & 76,0 & 0,930 \\
$\begin{array}{l}19 \text { - Desde que está em } \\
\text { tratamento, alguma vez } \\
\text { deixou de tomar sua } \\
\text { medicação um dia ou mais? }\end{array}$ & Não & 56,4 & - \\
$\begin{array}{l}\text { 20 - Utiliza alguma estratégia } \\
\text { para lembrar-se de tomar a } \\
\text { medicação? }\end{array}$ & Não & 67,6 & - \\
\hline
\end{tabular}

\section{DISCUSSÃO}

Neste estudo, foi possível identificar que 16,8\% das PVHIV apresentaram adesão estrita ao tratamento. Em pesquisa realizada com a finalidade de validar e adaptar o CEAT-VIH ao Brasil, a adesão estrita é observada em cerca de $20 \%$ dos pacientes, entretanto, destaca-se que em outros estudos brasileiros, utilizando o mesmo questionário, o percentual de adesão estrita, apresentou variação entre $14 \%$ 29,7\%e 48,7\%,11-13. Em outros países onde o CEAT-VIH também foi utilizado, e apesar de apresentarem características distintas, os resultados mostraram-se próximos ao deste estudo quanto à avaliação da adesão. Na Romênia e na Colômbia, a adesão estrita foi descrita em $15 \%$ da população estudada, todavia, no Peru, o percentual aumentou para $25,5 \%{ }^{14-16}$. 
Assim, por a adesão ser considerada um processo multifatorial, dinâmico e variável, e estar implicada a um conjunto de características e ações que podem ou não interferir na ingestão dos medicamentos antirretrovirais, ressalta-se a importância dos serviços de saúde em realizar constantemente a avaliação da adesão ${ }^{7}$.

Sugere-se a utilização do CEAT-VIH, uma vez que este instrumento é uma ferramenta eficiente que permite avaliação fundamentada em um caráter multidimensional, abrangendo os principais fatores determinantes da adesão baseado nas dimensões que ele contempla como a conformidade com o tratamento; os fatores que modulam a adesão; a interação entre profissional e paciente; relação quanto ao esforço e o tempo de tratamento; e a gravidade dos efeitos colaterais ${ }^{8}$.

A partir das correlações entre as perguntas da Tabela 01 foi possível identificar que estes itens estavam ligados diretamente com a ingesta do medicamento e aspectos psicossociais. Dessa forma, e apesar de haver correlação entre sentir-se melhor, pior, triste ou deprimido e deixar de tomar as medicações, observa-se que a maioria das PVHA não deixou de tomar sua medicação ao sentirem-se bem. Porém, caso haja algum tipo de efeito colateral, e o paciente se sentir pior, ele pode deixar de tomar seus medicamentos utilizando como explicação o prejuízo à sua saúde a partir da sua ingesta ${ }^{17}$.

A satisfação com o tratamento a partir da melhora da saúde leva à compreensão do benefício do uso dos medicamentos, proporcionando maior capacidade de seguir o tratamento. Sentir-se bem realizando a TARV pode ser visto como um aspecto facilitador da adesão, visto que as PVHIV referem não querer vivenciar a doença, e para tanto tentam seguir corretamente o tratamento. A partir do momento que tomam consciência que ao fazer uso correto dos medicamentos tem a possibilidade de aumentar sua sobrevida e viver com qualidade, sentemse motivados a cumprir o tratamento ${ }^{18}$.

Ainda, otimismo, expectativas ou crenças favoráveis em relação à TARV, com percepção da ausência de dificuldades e problemas relacionados ao tratamento, foram associados com maior grau de adesão. Promoção de esperança ou positividade ao tratamento pode ser importante, pois ao surgir dificuldades, perspectivas favoráveis e otimistas intensificam os esforços das pessoas para tornar regular o tratamento ${ }^{8}$.
Outro fator que dificulta a adesão é o fato de as pessoas realizaram esforços ou de até que ponto se sentem capazes de seguir o tratamento. As pessoas que necessitam realizar um esforço maior para realizar o tratamento acabam, por vezes, não o realizando adequadamente $^{16}$. Além disso, o apoio insuficiente do meio social e familiar parece afetar negativamente a adesão, encaminhando o paciente à depressão e à desesperança. Portanto, o investimento na atenção aos aspectos psicossociais, envolvendo a rede de apoio social desta população, tem papel fundamental em amenizar as consequências de eventos estressantes, pois estes fatores são relevantes do ponto de vista da qualidade de vida de PVHIV e, consequentemente, na adesão ao tratamento ${ }^{19}$.

A capacidade de seguir o tratamento, junto à satisfação do seu uso, remete à expectativa de autoeficácia que demonstra o quanto o paciente se julga capaz de seguir as prescrições da TARV. Essa expectativa muda de acordo com situações do cotidiano, em que o julgamento da capacidade de cumprir as prescrições pode vir a diminuir. Dessa forma, estas situações devem ser identificadas e trabalhadas pelos profissionais de saúde junto aos pacientes, a fim de auxiliar no manejo destas situações e potencializar a adesão $0^{20}$.

Por fim, constataram-se algumas limitações neste estudo quanto ao tamanho da amostra investigada, à restrição do cenário a um HU do interior do sul do país e à utilização de um questionário de coleta de dados com informações autorreferidas, ou seja, que não foram averiguadas em outras fontes de dados, o que pode ocasionar viés da informação coletada.

\section{CONCLUSÕES}

A baixa/inadequada adesão verificada em praticamente metade dos sujeitos desta pesquisa certifica o uso do CEAT-VIH quanto ferramenta oportuna a ser utilizada no dia a dia das equipes de saúde que atuam junto às PVHIV e necessitam realizar a avaliação de adesão de seus pacientes. $\mathrm{O}$ instrumento apresenta uma abordagem multidimensional, de fácil aplicação em que o enfermeiro e/ou demais profissionais de saúde podem utilizá-lo a fim de avaliar a adesão. 
Ter uma metodologia definida para essa avaliação promove o planejamento no processo do cuidado a fim de diagnosticar e intervir nas situações que interferem na adesão quando esta não for satisfatória.

Os resultados das correlações refletem os fatores que interferem na adesão como, por exemplo, deixar de tomar a medicação conforme está se sentindo no momento. Da mesma forma que identificar e potencializar o estímulo à boa adesão a partir do bom estado de saúde.

Deste modo, o planejamento das ações para o cuidado pode ser desencadeado também pelo enfermeiro durante as consultas de enfermagem, diagnosticando os fatores que afetam a adesão, e disseminado a equipe multiprofissional quando necessário.

\section{REFERÊNCIAS}

1. Costa JSD da, Victora CG. O que é "um problema de saúde pública”?. Rev. bras. epidemiol.. 2006;9(1):1446. Disponível em: http://dx.doi.org/10.1590/\$1415790X2006000100018. Acessado em: 08 Nov. 2017.

2. Souza BB, Vasconcelos CC, Tenório DM, Lucena MGA, Holanda RLT. A Política de AIDS no Brasil: uma abordagem histórica. J Manag Prim Health Care. 2010;1(1):23-6. Disponível em: http://www.jmphc. com.br/saude-publica/index.php/jmphc/article/ viewFile/90/91. Acesso em: 25 Out. 2017.

3. Montaner JSG, Lima VD, Harrigan PR, Lourenço L, YIPB, Nosyk B, Wood E, Kerr T, Shannon K, Moore D, Hogg RS, Barrios R, Gilbert M, Krajden M, Gustafson R, Daly P, Kendall P. Expansion of HAART coverage is associated with sustained decreases in HIV/AIDS morbidity, mortality and HIV transmission: the "HIV treatment as prevention" experience in a Canadian setting. PLoS ONE. 2014;9(2):e.87872. Disponível em: https://doi.org/10.1371/journal.pone.0087872. Acesso em: 25 Out. 2017.

4. Rio Grande Do Sul. Secretaria de Estado da Saúde. Departamento de Ações em Saúde. Seção Estadual de Controle das IST/Aids. Boletim Epidemiológico: HIV/ Aids. Porto Alegre. Secretaria de Estado da Saúde/ Escola de Saúde Pública; 2016. 96 p.
5. Mendes EV. O cuidado das condições crônicas na atenção primária à saúde: o imperativo da consolidação da estratégia da saúde da família. Brasília: Organização Pan-Americana da Saúde; 2012. $512 \mathrm{p}$.

6. Brasil. Ministério da Saúde. Secretaria de Vigilância em Saúde. Departamento de Vigilância, Prevenção e Controle das Infecções Sexualmente Transmissíveis, do HIV/Aids e das Hepatites Virais. Protocolo Clínico e Diretrizes Terapêuticas para Manejo da Infecção pelo HIV em Adultos. Brasília. Ministério da Saúde; 2017. 214 p.

7. Polejack L, Seidl EMF. Monitoramento e avaliação da adesão ao tratamento antirretroviral para HIV/ aids: desafios e possibilidades. Ciênc. saúde coletiva. 2010;15(supl 1):1201-8. Disponível em: http://dx.doi. org/10.1590/S1413-81232010000700029. Acesso em: 25 Out. 2017.

8. Remor E, Milner-Moskovics J, Preussler G. Adaptação brasileira do "Cuestionario para la Evaluación de la Adhesión al Tratamiento Antiretroviral". Rev. Saúde Pública. 2007;41(5):685-94. Disponível em: http:// dx.doi.org/10.1590/S0034-89102006005000043. Acesso em: 25 Out. 2017.

9. Remor E. Systematic review of the psychometric properties of the questionnaire to evaluate the adherence to HIV therapy (CEAT-VIH). Patient. 2013;6:61-73. Disponível em:https://doi.org/10.1007/ s40271-013-0009-0. Acesso em: 25 Out. 2017.

10. Bisquerra R, Sarriera JC, Martínez F. Introdução à estatística: enfoque informático com o pacote estatístico SPSS. Porto Alegre: Artmed; 2004. 255 p.

11. Galvão MTG, Soares LL, Pedrosa SC, Fiuza MLT, Lemos LA. Qualidade de vida e adesão à medicação antirretroviral em pessoas com HIV. Acta paul. enferm.. 2015;28(1):48-53. Disponível em: http:// dx.doi.org/10.1590/1982-0194201500009. Acesso em: 25 Out. 2017.

12. Moraes DCA, Oliveira RC, Motta MCS, Ferreira OLC, 
Andrade MS. Terapia antirretroviral: a associação entre o conhecimento e a adesão. Rev. pesqui cuid fundam. 2015;7(4):3563-73. Disponível em: http:// saudepublica.bvs.br/pesquisa/resource/pt/bde27211. Acesso em: 25 Out. 2017.

13. Silva ACO, Reis RK, Nogueira JA, Gir E. Quality of life, clinical characteristics and treatment adherence of people living with HIV/AIDS. Rev. Latino-Am. Enfermagem. 2014;22(6):994-1000. Disponível em: http://dx.doi.org/10.1590/0104-1169.3534.2508. Acesso em: 25 Out. 2017.

14. Dima AL, Schweitzer AM, Diaconita R, Remor E, Wanless RS. Adherence to ARV medication in Romanian Young adults: Self-reported behaviour and psychological barriers. Psychol Health Med. 2013;18(3):343-54. Disponível em: https://www.ncbi. nlm.nih.gov/pubmed/22985131. Acesso em: 25 Out. 2017.

15. Remor E. Self-Reported Adherence to Antiretroviral Therapy in HIV+ Colombian Population. SAGE Open. 2013;3(3):1-7. Disponível em: https://doi. org/10.1177/2158244013497727. Acesso em: 25 Out. 2017.

16. Tello-Velásquez JR, Díaz-Llanes BE, Mezones-Holguín E, Rodríguez-Morales AJ, Huamaní C, Hernández AV, Arévalo-Abanto J. La mala calidad de sueño se asocia a uma menor adherencia al tratamiento antirretroviral de gran actividad em pacientes peruanos com infección por VIH/SIDA. Cad saúde pública. 2015;31(5):989-1002. Disponível em: http:// dx.doi.org/10.1590/0102-311X00010014. Acesso em: 25 Out. 2017.

17. Fiuza MLT, Lopes EM, Alexandre HO, Dantas PB, Galvão MTG, Pinheiro AKB. Adesão ao tratamento antirretroviral: assistência integral baseada no modelo de atenção às condições crônicas. Esc. Anna Nery. 2013;17(4):740-8. Disponível em: http:// dx.doi.org/10.5935/1414-8145.20130019. Acesso em: 25 Out. 2017.

18. Vizeu Camargo B, Bousfield ABS, Giacomozzi AI,
Papaleo Koelzer L. Representações sociais e adesão ao tratamento antirretroviral. liber.. 2014;20(2):22938. Disponível em: http://www.scielo.org.pe/ scielo.php?script $=$ sci_arttext $\&$ pid $=$ S1729. $48272014000200004 \& \operatorname{lng}=$ es\&nrm $=$ iso. Acesso em: 25 Out. 2017.

19. Calvetti P, Giovelli GRM, Gauer GJC, Moraes JFD. Níveis de Ansiedade, Estresse Percebido e Suporte Social em Pessoas que Vivem com HIV/Aids.Psic.: Teor. e Pesq.. 2016;32(4):e324317. Disponível em: http://dx.doi.org/10.15900102.3772e324317. Acesso em: 25 Out. 2017.

20. Santos EEP, Padoin SMM, Zuge SS, Schwarzbold AV, Magnago TSBS, Paula CC. Expectativa de autoeficácia para tratamento de adultos com AIDS em um hospital universitário. Rev enferm UFPE. 2014;8(8):27972804. Disponível em: http://www.revista.ufpe. br/revistaenfermagem/index.php/revista/article/ view/5438/pdf_5929. Acesso em: 25 Out. 2017.

Recebido em: 18/11/2017 Aceito em: 25/05/2018 